\title{
1 Determining protein structures using 2 deep mutagenesis
}

3 Jörn M. Schmiedel ${ }^{1}$, Ben Lehner ${ }^{1-3^{*}}$

$4{ }^{1}$ Centre for Genomic Regulation (CRG), The Barcelona Institute of Science and Technology,

5 Dr. Aiguader 88, Barcelona 08003, Spain

$6{ }^{2}$ Universitat Pompeu Fabra (UPF), Barcelona 08003, Spain

$7 \quad{ }^{3}$ ICREA, Pg. Lluís Companys 23, 08010 Barcelona, Spain

$8 \quad$ *email: ben.lehner@crg.eu

\section{Abstract}

11 Determining the three-dimensional (3D) structures of macromolecules is a major goal of 12 biological research because of the close relationship between structure and function but 13 thousands of protein domains still have unknown structures. Structure determination usually 14 relies on physical techniques including x-ray crystallography, NMR spectroscopy and cryo15 electron microscopy. Here we present a method that allows the high-resolution 3D backbone 16 structure of a biological macromolecule to be determined only from measurements of the activity 17 of mutant variants of the molecule. This genetic approach to structure determination relies on 18 the quantification of genetic interactions (epistasis) between mutations and the discrimination of 19 direct from indirect interactions. This provides an alternative experimental strategy for structure 20 determination, with the potential to reveal functional and in vivo structural conformations. 


\section{Introduction}

23 Despite years of effort and technological development, thousands of protein domains still have unknown 3D structures ${ }^{1}$. Mutations within a protein or RNA can have non-independent effects on fitness ${ }^{2-5}$ and double mutants have been used to probe the energetic couplings between positions in a protein to understand determinants of protein folding and stability ${ }^{6,7}$. Early work revealed that at least some strongly interacting positions within a protein are in direct structural contact $^{6-10}$ (Fig. 1a). Deep mutational scanning (DMS) of proteins ${ }^{11-14}$ and RNAs ${ }^{15-18}$ has further revealed that some - but by no means all - genetic (or epistatic) interactions occur between structurally proximal mutations.

Support for the idea that non-independence between mutations provides structural information comes from the analysis of amino acid and nucleotide sequence evolution. Here, correlated pairs of amino acids or nucleotides in multiple sequence alignments identify co-evolving positions within proteins and RNAs ${ }^{19-21}$. These patterns of co-evolution have been used to identify energetically coupled positions and independently evolving 'sectors' within proteins ${ }^{22,23}$. Moreover, when very large numbers of homologous proteins and RNAs are avaiable in sequence databases, the application of global statistical models can discriminate direct structural contacts from patterns of co-evolution ${ }^{24-26}$, allowing the prediction of macromolecular structures and interactions s, $^{1,27-35}$.

Could epistatic interactions quantified from DMS experiments be used to determine macromolecular structures? If successful, structure determination by DMS would offer a number of advantages over established techniques. First, it requires no specialized equipment or expertise beyond the ability to mutate a molecule, select functional variants, and quantify enrichments by sequencing. Appropriate in vitro and in vivo selection assays already exist for many molecules of interest and generic assays based on folding, stability, and physical interactions have also been developed ${ }^{11,36-39}$. Second, it could be applied to molecules whose structures are difficult to determine by physical techniques such as intrinsically disordered and membrane proteins. Third, unlike evolutionary coupling analysis there is no requirement for large numbers of homologous sequences and so it could be applied to fast-evolving, recentlyevolved and de novo designed proteins and RNAs ${ }^{1,28,40}$. Finally, and perhaps most importantly,

51 it would provide a general strategy to determine the physiologically relevant structures of molecules whilst they are performing particular functions that can be selected for, including in 
53 vivo within cells. A potentially cheap and straightforward approach for studying macromolecular

54 structures in vivo would be an exciting new frontier for cell and molecular biology.

55 Here we show that DMS of proteins can provide sufficient information to determine their high56 resolution 3D backbone structures. Our statistical approach quantifies how often mutations 57 between positions interact epistatically and how these epistatic interaction patterns correlate. 58 These metrics accurately identify individual tertiary structure contacts as well as secondary 59 structure elements within a protein. The same approach also identifies contacts between protein 60 interaction partners. DMS data alone suffice to determine protein structures with accuracies 61 down to $1.9 \AA$ Ca root mean square deviation (RMSD) compared to known reference structures. 62 Moreover, we show that deep learning can further improve prediction performance, allowing the 63 use of sparser and lower quality DMS datasets for structure determination. Our approach 64 therefore provides an experimental strategy for structure determination that can reveal 65 functional and in vivo structural conformations. 


\section{Results}

\section{Epistasis is enriched in but not exclusive to structural contacts}

68 We first investigated the relationship between epistasis and structure for more than half a million

69 mutant variants $(55 \times 19=1,045$ single mutants plus nearly $55 \times 54 \div 2 \times 19 \times 19=536,085$

70 double mutants) of the protein G B1 domain $(\mathrm{GB} 1)^{13}$. For these variants, protein fitness was

71 quantified using binding to an immunoglobulin $G$ fragment as a selection assay, resulting in a

72 two orders of magnitude measurement range with a median relative error of fitness estimates of

$73 \quad 2.8 \%$ (Supplementary Fig. 1a, Table 1).

74 We used a running median surface approach as null model for the independence of double

75 mutation effects (Fig. 1b) to account for non-specific dependencies between mutants introduced

76 by the fitness assay or non-specific epistatic behavior from thermodynamic stability effects ${ }^{2,11}$.

77 Double mutants were classified as positive or negative epistatic if they have more extreme

78 fitness than the $95^{\text {th }}$ or $5^{\text {th }}$ percentile fitness surfaces, respectively. Restricting the classification

79 of epistasis to variants not impeded by measurement errors resulted in $80 \%$ and $55 \%$ of double

80 mutants being suitable for positive or negative epistasis classification, respectively, with

81 substantial variability across the position matrix (Supplementary Fig. 1b-f and Table 1).

82 Consistent with previous observations ${ }^{12-14}$, both positive and negative epistatic double mutants

83 are enriched for proximal variants, for example, more than 2-fold at $8 \AA$ distance (Fig. 1c, only

84 considering position pairs separated by more than 5 amino acids (aa) in the linear sequence;

85 closer positions are trivially also close in 3D space, and their proximity contributes little to

86 successful structure prediction ${ }^{30}$ ). However, about $75 \%$ of epistatic interactions are between

87 positions that are not in direct contact in the protein (as judged by an $8 \AA$ distance cutoff),

88 suggesting that indirect effects often underlie specific epistatic interactions within a

89 molecule ${ }^{22,23}$. The challenge for structure determination therefore becomes how to infer direct

90 structural contacts from the mixture of direct and indirect effects that underlie epistasis.

91 Likelihood of epistatic interactions and correlated interaction

92 profiles predict tertiary structure contacts

93 To discriminate direct structural contacts from a list of thousands of epistatic double mutants we

94 used two measures. 
The first, which we refer to as the enrichment score, quantifies how often double mutants between each pair of positions interact with positive or negative epistasis (Fig. 2a). Calculating the fraction of epistatic interactions separately for either positive or negative interactions enriches for structural contacts, but for different regions of the domain (Fig. 2b, Supplementary Fig. 2). Combining the positive and negative epistatic fractions, taking into account quantification errors, further enriches for direct contacts (positive predictive value for top L/2 contacts $\mathrm{PPV}_{\mathrm{L} / 2}=61 \%, \mathrm{PPV}_{\mathrm{L}}=60 \%$, with $\mathrm{L}=55$ as the length of the mutated sequence, Fig. $2 \mathrm{~g}$ ), with these contacts evenly distributed across the domain (Fig. 2b,f).

103 The second score, which we refer to as the correlation score, quantifies the similarities of 104 epistasis interaction profiles - how a position interacts with all other positions in the protein 105 between each pair of positions. The assumption underlying this score is that positions close in 106 space in a structure should interact similarly with all other positions (Fig. 2c). We used partial 107 correlations - thus correcting correlations for transitive signals - to better distinguish direct from 108 indirect contacts and again calculated scores separately for positive and negative interactions 109 before merging them taking into account quantification errors (Fig. 2d). The final correlation 110 scores show a more binary all-or-none relationship with distance than the enrichment scores or 111 when using simple correlations to quantify similarity (Fig. 2e), thus better prioritizing the top direct structural contacts across the whole domain (Fig. 2f,g, $\mathrm{PPV}_{\mathrm{L} / 2}=79 \%, \mathrm{PPV} \mathrm{L}=60 \%$ ).

113 Finally, combining the enrichment and correlation scores into a combined score by simply 114 summing normalized scores further improves contact predictions, especially when considering 115 lower ranked predictions $\left(\mathrm{PPV}_{\mathrm{L} / 2}=82 \%, \mathrm{PPV}=73 \%\right.$, Fig. $\left.2 \mathrm{~g}\right)$.

\section{Identification of secondary structure elements}

117 We hypothesized that the periodic geometrical arrangement of aa residues in secondary 118 structures (3.6 residues per alpha-helical turn and alternating side-chain directions in beta 119 strands) might result in periodic epistasis patterns in DMS data (Fig. 3a) $)^{28,41}$. We used a two120 dimensional (2D) kernel smoothing approach to detect alpha helical and beta strand 121 periodicities (Fig. 3b) and found significant periodicities for an alpha helix and four beta strands 122 that coincide very well with secondary structure elements in the reference structure (Fig. 3c and 123 Supplementary Fig. 3a). Moreover, stretches of off-diagonal, long-distance interactions show 124 the expected alternating patterns for either parallel or anti-parallel beta sheets, with the top 125 predictions corresponding to the known anti-parallel interactions of $\beta 1-\beta 2$ and $\beta 3-\beta 4$ as well 126 as the parallel interaction of $\beta 1-\beta 4$ (Fig. $3 d$ and Supplementary Fig. 3b,c). Furthermore, 
updating beta strand predictions according to inferred beta sheet pairings led to improved beta strand prediction, notably enforcing a split between $\beta 1$ and $\beta 2$ and correcting the length of $\beta 3$ and $\beta 4$ (Fig. 3c,d). Overall, these secondary structure element predictions achieve precision and recall values of about $90 \%$ when derived from correlation scores (or combined scores, Supplementary Fig. 3d). Predictions from enrichment scores are less precise, thus suggesting that eliminating transitive, indirect interactions is important for secondary structure prediction.

\section{Tertiary structure prediction}

We next tested whether the DMS data alone could be used to determine the structure of the protein domain. We performed structural simulations by simulated annealing using the XPLOR$\mathrm{NIH}$ modeling suite ${ }^{42}$, having as structural restraints the top $\mathrm{L}$ scoring position pairs as well as dihedral angle restraints for predicted secondary structure elements and restrictive distance restraints for predicted beta sheet positions that form hydrogen bonds (Fig. 3e).

Comparing the structural models against the experimentally determined crystal structure of GB1 revealed that the combined scores provided the best predictions, with the top $5 \%$ of models (25/500, evaluated on internal energy terms) having an average C $\alpha$-root mean squared deviation $(\langle C \alpha-R M S D\rangle)$ of $1.9 \AA$ and an average template modeling score of 0.71 (Fig. $3 \mathrm{f}, \mathrm{g}$ and Supplementary Fig. 3f), which is very close to the optimum achievable with our simulation protocol (using contacts, secondary structure elements and beta sheet interactions from the reference structure, $\langle C \alpha-R M S D\rangle=1.4 \AA$ and TM score $=0.8)$. Consistent with the somewhat lower precision of contact and secondary structure predictions, models generated with restrains from enrichment or correlation scores have on average a lower accuracy $(\langle C \alpha-R M S D\rangle=3.4 \AA$ and $\langle C \alpha-R M S D\rangle=2.6 \AA$, respectively), with correlation score models, however, performing consistently better.

Together, this shows that DMS alone is sufficient to accurately determine the backbone structure of a protein domain.

\section{Deep mutagenesis identifies protein interaction contacts and} structures

Epistatic interactions can also occur between different proteins, for example between physical interaction partners ${ }^{3}$. We tested whether epistasis between two proteins quantified using our metrics could predict their structural interactions. We used a dataset ${ }^{11}$ in which we had made all 
possible aa mutations at 32 positions in the products of the FOS and JUN proto-oncogenes and quantified the physical interaction of all single and (trans-)double mutants using a deep sequencing-based protein complementation assay (Fig. 4a, Table 1). Notably, enrichment scores show a binary all-or-none relationship with distance similar to the correlation scores in GB1 (Fig. 4b), with distant position pairs across the interaction surface contained in a low enrichment score peak and proximal interactions enriched for high enrichment scores. Indeed, the top 11 enrichment score pairs are all proximal interactions, and the precision of contact prediction is $\mathrm{PPV}_{\mathrm{L} / 2}=75 \%$ and $\mathrm{PPV}_{\mathrm{L}}=66 \%$ (12-fold and 10.5-fold over expectation). Moreover, top enrichment score pairs are evenly distributed across the interaction surface (Fig. 4a,c).

Correlating the epistatic interaction profiles between columns of the epistatic enrichment matrices compares the epistatic interactions that two positions in FOS have with all positions in JUN. Therefore, the similarity of column-wise epistatic profiles identifies the cis relationships between positions in FOS, while row-wise interaction profiles identify cis relationships between positions in JUN (Supplementary Fig. 4a). The cis-interaction maps from correlation scores for both FOS and JUN are highly enriched for strong local interactions and applying our secondary structure prediction algorithms reveals strong alpha helix propensities across the full lengths of both FOS and JUN, consistent with the coiled-coil structure of the complex (Fig. 4c and Supplementary Fig. 4b).

This shows that DMS of protein interaction partners can accurately predict direct contacts across the interaction surface as well as reveal the underlying structural conformations of the interaction partners themselves.

\section{Generality and data requirements for successful protein structure}

\section{prediction}

To test the generality of our approach, we analyzed two additional DMS of individual protein domains, the Pab1 RRM2 domain ${ }^{12}$ and the hYAP65 WW domain ${ }^{43}$ (Fig. 5a,b). These datasets contain only incomplete sets of double mutants $(\sim 10 \%)$, were sequenced less deeply and have up to six times smaller measurement range, resulting in up to three-times higher relative measurement errors and fewer double mutants suitable for quantification of epistasis (especially negative epistasis) (Supplementary Fig. 5a, Table 1). Nonetheless, tertiary contacts can be predicted with good precision (combined score $\mathrm{PPV}_{\mathrm{L} / 2}=57 \%$ (3-fold higher than random expectation) and $\mathrm{PPV}_{\mathrm{L} / 2}=59 \%$ (3.9-fold over expectation) for RRM2 and WW domain, respectively; Fig. 5c,d and Supplementary Fig. 5b). Secondary structure predictions were 
inaccurate and underpowered ( $0 \%$ precision), but beta sheet pairing was inferred correctly (100\% precision and recall for RRM domain), albeit off by one and two positions for the two antiparallel sheet interactions in the WW domain (Fig. 5c,d).

We used the top L/2 predicted combined score contacts to model the structure of the secondary structure-rich central part of the WW domain (positions 6 to 29, 24 amino acids, see Methods). The top $5 \%$ of structural models have an average accuracy of $3.3 \AA\langle C \alpha-R M S D\rangle$ compared to the reference structure (Fig. 5a), which is on par with simulations using a set of 'true' contacts $(\langle C \alpha-R M S D\rangle=3.6 \AA$ ) (Supplementary Fig. $5 \mathrm{c}$ ). We could not make structural predictions for the RRM domain because it was mutagenized in three independent segments.

To estimate the minimal requirements for DMS datasets to be useful for structure prediction, we investigated how robust our prediction strategy is to changes in data quality by artificially downsampling the GB1 domain dataset.

First, we considered the sequencing read coverage and find that even using only $10 \%$ of the 600 million sequencing reads in the full GB1 dataset hardly affects the precision of predicted tertiary contacts $\left(P P V_{L}=64 \%\right.$, a drop by $9 \%$ compared to the full dataset, Fig. 5e). Only when using just $2.5 \%$ of sequencing reads ( 15 million) does the precision of the top $L$ contacts drop below $50 \%\left(P P V_{L}=45 \%\right)$.

Second, we simulated a 'doped' mutagenesis dataset, by only considering amino acid mutations that can be reached by one mutation in the nucleotide sequence - thus reducing the coverage of double mutants to $\sim 10 \%$ (similar to the RRM and WW domain datasets). The doped dataset exhibits a decrease in precision of predicted tertiary contacts of about $20 \%\left(P P V_{L}=51 \%\right.$, Fig. $5 \mathrm{e})$. Moreover, the doped dataset shows an increased sensitivity to lower sequencing read coverage.

Third, we tested the effect of small signal-to-noise ratios (i.e. the measurement range of the selection assay relative to the median error of fitness estimates, which results in nonquantifiably of negative epistasis, see Supplementary Figs. $1 d-f$ and $5 a$ ), by using only positive epistasis information to calculate interaction scores. This also results in a drop of precision of about $20 \%\left(P P V_{L}=55 \%\right)$. In contrast, only using negative epistasis information resulted in a drop to $33 \%$ precision, as low as a doped dataset with low sequencing coverage.

Finally, we evaluated how differences in prediction performance of tertiary contacts affect structural modeling. Changes in accuracy of the top structural models scale with changes in 
contact prediction performance (Fig. 5f). Down-sampling of sequencing reads in the complete dataset from $100 \%$ to $2.5 \%$ leads to a decrease in accuracy from $2.5 \AA$ to $4 \AA\langle C \alpha-R M S D\rangle$,

222 which is roughly also the accuracy of top structural models from the doped dataset and the 223 dataset using only positive epistasis information.

224 Together, these results support the generality of our approach for extracting structural 225 information from DMS data, including from sparser and lower quality datasets.

\section{Deep learning improves contact prediction}

227 Evolutionary coupling-based structural predictions have been successfully improved by machine 228 learning approaches that transform the 2D interaction score maps after learning the 229 stereotypical patterns between evolutionary coupling-predicted contact maps and 230 experimentally determined contact maps ${ }^{44,45}$.

231 We tested whether machine learning can also improve DMS-derived contact predictions. We 232 applied a convolutional neural network called DeepContact, developed by Liu et al. ${ }^{44}$, which 233 transforms a 2D interaction score map based on the structural patterns it has previously learned 234 on evolutionary coupling-derived contact predictions for representative families of the SCOPe 235 database $^{46}$ (Fig. 6a and Methods).

236 We first transformed the GB1 domain combined score interaction map with the DeepContact 237 network. These transformations take as sole input our DMS-derived predictions and include no 238 evolutionary coupling or otherwise-derived structural predictors for GB1. The scores on the 239 transformed map are much less noisy, with high scores exclusively focused in areas of 240 structural contacts, especially those of secondary structure element interactions, and areas 241 devoid of structural contacts showing homogenously low scores (Fig. 6b). The precision of top 242 predicted contacts improves from $82 \%$ to $96 \%$ for $L / 2$ and from $73 \%$ to $87 \%$ for $L$ predicted 243 contacts (Fig. 6c).

244 Predictions derived from the two other GB1 interaction scores (enrichment and correlation 245 scores) as well as the interaction score maps for the other datasets (downsampled GB1, FOS246 JUN, RRM, WW1) show similar improvements both in terms of cleaner interaction score maps 247 that better resemble the reference contact maps as well as increases in contact prediction 248 performance of up to $30 \%$ (Supplementary Fig. 6). In contrast, randomized interaction score 249 maps show no changes in prediction performance over random expectation after transformation 250 with DeepContact (Fig. 6c). 
251 Finally, we tested whether DeepContact-transformed contact predictions could also improve 252 structural modeling. On down-sampled GB1 datasets, DeepContact-transformed predictions 253 increased the accuracy of structural models by up to $2.6 \AA$ (Fig. 6d). For the complete datasets 254 with only $25 \%$ or $10 \%$ of sequencing reads, the top structural models have better accuracy than 255 those from the complete dataset with full sequencing read coverage but untransformed scores. 256 Also, structural models based on DeepContact-transformed scores from the doped dataset with 257 full or $25 \%$ sequencing coverage and those from the dataset using only positive epistasis 258 information reach average accuracies of $3.2 \AA\langle C \alpha-R M S D\rangle$. Only for the two datasets with $2592.5 \%$ sequencing read coverage do structural simulations based on DeepContact-transformed 260 scores not improve model accuracy.

261 This shows that machine learning can substantially improve contact map prediction from DMS 262 data, thus allowing the use of even sparser and lower quality data for accurate structure 263 prediction.

\section{Discussion}

265 We have shown here that simply quantifying the activity of a large number of single and double mutant variants of a macromolecule can provide enough information to reliably determine its 3D 267 fold.

268 Our analyses and previous work ${ }^{6-9,11-18}$ have shown that many epistatic interactions occur 269 between positions that are not in direct structural contact. Indeed, in the protein G B1 domain, 270 the interactions are strikingly modular, with two mutually exclusive clusters of positive and 271 negative epistatic interactions arising potentially from differential energetic couplings to protein 272 stability and binding (Fig. 2b,d and Supplementary Fig. 2c), somewhat reminiscent of the 273 concept of semi-independent energetically coupled protein sectors identified from patterns of 274 sequence co-evolution ${ }^{22,23}$.

275 Nonetheless, aggregating epistatic interactions on position pairs, merging of positive and 276 negative epistasis information and partial correlation analysis of epistasis interaction profiles can 277 successfully discriminate direct from indirect structural contacts. Thus, mostly indirect epistatic 278 couplings can be transformed to accurately predict secondary structure elements and tertiary 279 contacts to reveal the protein fold. 
We have shown that our approach works robustly across multiple protein domains and a protein interaction. Moreover, we have demonstrated that the application of a convolutional neural network previously trained on patterns of co-evolution in proteins of known structure both improves structure prediction and allows the use of much lower quality DMS datasets. We note that our approach is likely to be only one of several that could work ${ }^{47}$.

We expect that development of the computational approach (consideration of the underlying physico-chemistry, better scoring methods, and extracting side-chain information) as well as integration with other structural predictors ${ }^{44,48,49}$ and homology-driven structure modeling ${ }^{50,51}$ is likely to further improve accuracy and lower the data quality requirements for structure determination by deep mutagenesis.

Will it be possible to determine the structures of larger molecules by deep mutagenesis? It is currently unclear how the requirements for variant coverage scale with protein length or the complexity of folds. However, the fact that sparse double mutant datasets can suffice for structure prediction and the rapid development of DNA synthesis and sequencing technologies suggest that similar approaches may work for larger structures. Currently, DMS libraries for larger proteins could be created via fragment-based ligation ${ }^{52}$ or programmed mutagenesis ${ }^{53,54}$ and sequenced by linking variants to short barcodes ${ }^{36,37}$ to overcome the current size limitations of short-read sequencers.

A limitation of the current approach is that, similar to methods based on evolutionary couplings of residues ${ }^{24,30}$, it identifies tertiary contacts but does not provide atom-level information about side-chain orientations. However, our finding that epistatic interactions contain information on the periodic arrangement of side-chain orientations in secondary structure elements and that tertiary contacts are better described by side-chain than backbone atom distances (Supplementary Fig. 7) suggests that genetic interactions are mostly mediated by structural interactions of amino acid side-chains and that it might be possible to extract additional information about their orientations.

Determining structures by DMS offers several practical advantages. The approach does not require the expensive scientific infrastructure of physical techniques and uses methods familiar to most molecular biologists. Selection assays based on known functions or interaction partners already exist for many proteins ${ }^{13,16,17,43,52,55-59}$ and the development of generic assays for stability 310 and activity ${ }^{36-39}$ should allow it to be applied to molecules of unknown function. The approach 311 also potentially brings the power of high-throughput genomics to structural biology. For 
312 example, using the existing infrastructure of genomics institutes, a large-scale project to 313 systematically determine the structures of all protein domains of unknown structure is a 314 plausible endeavor. Finally, and perhaps most interestingly, DMS allows the structures of 315 macromolecules to be studied in vivo in the cell ${ }^{60}$. Ultimately, it is the structure of 316 macromolecules as they perform a particular function in vivo that are most of interest. Deep 317 mutagenesis, selection and sequencing provide a generic approach for 'in vivo structural 318 biology'.

319 In summary, DMS provides an experimental strategy for structure determination and opens up 320 the possibility of low cost and high-throughput determination of in vivo macromolecular 321 structures, both by individual laboratories and by large-scale genomics projects. 


\section{Acknowledgements}

323 We are grateful to Yang Liu and Jian Peng for making their DeepContact code available and for 324 their advice. We thank members of the Lehner laboratory, T. Gross, G. Mönke, M. Bolognesi 325 and C. Camilloni, for discussions and feedback. This work was supported by a European 326 Research Council (ERC) Consolidator grant (616434), the Spanish Ministry of Economy, 327 Industry and Competitiveness (MEIC; BFU2017-89488-P), the AXA Research Fund, the 328 Bettencourt Schueller Foundation, Agencia de Gestio d'Ajuts Universitaris i de Recerca 329 (AGAUR, 2017 SGR 1322), the EMBL-CRG Systems Biology Program, and the CERCA 330 Program/Generalitat de Catalunya. J.M.S. was supported by an EMBO Long-Term Fellowship 331 (ALTF 857-2016). This project has received funding from the European Union's Horizon 2020 332 research and innovation program under the Marie Skłodowska-Curie grant agreement 752809 333 (J.M.S). The authors acknowledge support from the Spanish Ministry of Economy, Industry and 334 Competitiveness (MEIC) to the EMBL partnership and the Centro de Excelencia Severo Ochoa.

\section{Author Contributions}

337 Conceptualization, J.M.S. and B.L.; Methodology, J.M.S.; Investigation, J.M.S.; Writing, J.M.S. 338 and B.L.; Supervision, B.L.

341 The authors declare no competing interests.

\section{Additional Information}

344 Correspondence and requests for materials should be addressed to B.L. 


\section{References}

3471 Ovchinnikov, S. et al. Protein structure determination using metagenome sequence data. $348 \quad$ Science 355, 294-298, doi:10.1126/science.aah4043 (2017).

3492 Tokuriki, N. \& Tawfik, D. S. Stability effects of mutations and protein evolvability. Current $350 \quad$ Opinion in Structural Biology 19, 596-604, doi:10.1016/j.sbi.2009.08.003 (2009).

3513 Lehner, B. Molecular mechanisms of epistasis within and between genes. Trends in $352 \quad$ Genetics : TIG 27, 323-331, doi:10.1016/j.tig.2011.05.007 (2011).

3534 Fowler, D. M. \& Fields, S. Deep mutational scanning: a new style of protein science. Nature Methods 11, 801-807, doi:10.1038/nmeth.3027 (2014).

Starr, T. N. \& Thornton, J. W. Epistasis in protein evolution. Protein science 25, 1204-

6 Horovitz, A. \& Fersht, A. R. Strategy for analysing the co-operativity of intramolecular interactions in peptides and proteins. $J$ Mol Biol 214, 613-617, doi:10.1016/00222836(90)90275-Q (1990).

$7 \quad$ Carter, P. J., Winter, G., Wilkinson, A. J. \& Fersht, A. R. The use of double mutants to detect structural changes in the active site of the tyrosyl-tRNA synthetase (Bacillus stearothermophilus). Cell 38, 835-840 (1984). pairwise interactions in the alpha-neurotoxin-nicotinic acetylcholine receptor complex through double mutant cycles. J Biol Chem 273, 10958-10964 (1998).

Chen, J. \& Stites, W. E. Energetics of side chain packing in staphylococcal nuclease assessed by systematic double mutant cycles. Biochemistry 40, 14004-14011 (2001).

Roisman, L. C., Piehler, J., Trosset, J. Y., Scheraga, H. A. \& Schreiber, G. Structure of the interferon-receptor complex determined by distance constraints from double-mutant cycles and flexible docking. Proceedings of the National Academy of Sciences 98, 13231-13236, doi:10.1073/pnas.221290398 (2001).

Diss, G. \& Lehner, B. The genetic landscape of a physical interaction. eLife 7, 594, doi:10.7554/eLife.32472 (2018). scanning of an RRM domain of the Saccharomyces cerevisiae poly(A)-binding protein. RNA 19, 1537-1551, doi:10.1261/rna.040709.113 (2013). epistasis throughout an entire protein domain. Current biology 24, 2643-2651, doi:10.1016/j.cub.2014.09.072 (2014).

14 Sahoo, A., Khare, S., Devanarayanan, S., Jain, P. C. \& Varadarajan, R. Residue proximity information and protein model discrimination using saturation-suppressor mutagenesis. eLife 4, 371, doi:10.7554/eLife.09532 (2015).

5 Li, C. \& Zhang, J. Multi-environment fitness landscapes of a tRNA gene. Nature Ecology \&; Evolution 15, 1, doi:10.1038/s41559-018-0549-8 (2018).

16 Li, C., Qian, W., Maclean, C. J. \& Zhang, J. The fitness landscape of a tRNA gene. Science 352, 837-840, doi:10.1126/science.aae0568 (2016). 
Domingo, J., Diss, G. \& Lehner, B. Pairwise and higher-order genetic interactions during the evolution of a tRNA. Nature 558, 117-121, doi:10.1038/s41586-018-0170-7 (2018).

Puchta, O. et al. Network of epistatic interactions within a yeast snoRNA. Science $\mathbf{3 5 2}$, 840-844, doi:10.1126/science.aaf0965 (2016).

Göbel, U., Sander, C., Schneider, R. \& Valencia, A. Correlated mutations and residue contacts in proteins. Proteins 18, 309-317, doi:10.1002/prot.340180402 (1994).

Altschuh, D., Lesk, A. M., Bloomer, A. C. \& Klug, A. Correlation of co-ordinated amino acid substitutions with function in viruses related to tobacco mosaic virus. Journal of molecular biology 193, 693-707 (1987).

21 Gloor, G. B., Martin, L. C., Wahl, L. M. \& Dunn, S. D. Mutual information in protein multiple sequence alignments reveals two classes of coevolving positions. Biochemistry 44, 7156-7165, doi:10.1021/bi050293e (2005).

Halabi, N., Rivoire, O., Leibler, S. \& Ranganathan, R. Protein sectors: evolutionary units of three-dimensional structure. Cell 138, 774-786, doi:10.1016/j.cell.2009.07.038 (2009).

Lockless, S. W. \& Ranganathan, R. Evolutionarily conserved pathways of energetic connectivity in protein families. Science 286, 295-299 (1999).

24 Morcos, F. et al. Direct-coupling analysis of residue coevolution captures native contacts across many protein families. Proceedings of the National Academy of Sciences 108, E1293-1301, doi:10.1073/pnas.1111471108 (2011).

25 Weigt, M., White, R. A., Szurmant, H., Hoch, J. A. \& Hwa, T. Identification of direct residue contacts in protein-protein interaction by message passing. Proceedings of the National Academy of Sciences 106, 67-72, doi:10.1073/pnas.0805923106 (2009).

Burger, L. \& van Nimwegen, E. Disentangling Direct from Indirect Co-Evolution of Residues in Protein Alignments. PLoS Computational Biology 6, e1000633, doi:10.1371/journal.pcbi.1000633 (2010).

28 Tóth-Petróczy, A. et al. Structured States of Disordered Proteins from Genomic Sequences. Cell 167, 158-170.e112, doi:10.1016/j.cell.2016.09.010 (2016).

29 Hopf, T. A. et al. Three-Dimensional Structures of Membrane Proteins from Genomic Sequencing. Cell 149, 1607-1621, doi:10.1016/j.cell.2012.04.012 (2012).

30 Marks, D. S. et al. Protein 3D structure computed from evolutionary sequence variation. PLOS ONE 6, e28766, doi:10.1371/journal.pone.0028766 (2011).

31 Jones, D. T., Buchan, D. W. A., Cozzetto, D. \& Pontil, M. PSICOV: precise structural contact prediction using sparse inverse covariance estimation on large multiple sequence alignments. Bioinformatics 28, 184-190, doi:10.1093/bioinformatics/btr638 (2012).

32 De Leonardis, E. et al. Direct-Coupling Analysis of nucleotide coevolution facilitates RNA secondary and tertiary structure prediction. Nucleic Acids Research 43, 10444-10455, doi:10.1093/nar/gkv932 (2015).

33 Sułkowska, J. I., Morcos, F., Weigt, M., Hwa, T. \& Onuchic, J. N. Genomics-aided structure prediction. Proceedings of the National Academy of Sciences 109, 1034010345, doi:10.1073/pnas.1207864109 (2012). 
34 Ovchinnikov, S. et al. Large-scale determination of previously unsolved protein structures using evolutionary information. eLife 4, e09248, doi:10.7554/eLife.09248 (2015).

35 Ovchinnikov, S., Kamisetty, H. \& Baker, D. Robust and accurate prediction of residueresidue interactions across protein interfaces using evolutionary information. eLife 3, e02030, doi:10.7554/eLife.02030 (2014).

Matreyek, K. A. et al. Multiplex assessment of protein variant abundance by massively parallel sequencing. Nature Genetics 50, 874-882, doi:10.1038/s41588-018-0122-z (2018).

Weile, J. et al. A framework for exhaustively mapping functional missense variants. Molecular Systems Biology 13, 957, doi:10.15252/msb.20177908 (2017).

Rocklin, G. J. et al. Global analysis of protein folding using massively parallel design, synthesis, and testing. Science 357, 168-175, doi:10.1126/science.aan0693 (2017).

Kim, I., Miller, C. R., Young, D. L. \& Fields, S. High-throughput analysis of in vivo protein stability. Molecular \& Cellular Proteomics : MCP 12, 3370-3378, doi:10.1074/mcp.0113.031708 (2013).

42 Schwieters, C. D., Kuszewski, J. J., Tjandra, N. \& Clore, G. M. The Xplor-NIH NMR molecular structure determination package. Journal of magnetic resonance 160, 65-73 (2003).

43 Araya, C. L. et al. A fundamental protein property, thermodynamic stability, revealed solely from large-scale measurements of protein function. Proceedings of the National Academy of Sciences 109, 16858-16863, doi:10.1073/pnas.1209751109 (2012).

44 Liu, Y., Palmedo, P., Ye, Q., Berger, B. \& Peng, J. Enhancing Evolutionary Couplings with Deep Convolutional Neural Networks. Cell Systems, doi:10.1016/j.cels.2017.11.014 (2017).

45 Schaarschmidt, J., Monastyrskyy, B., Kryshtafovych, A. \& Bonvin, A. M. J. J. Assessment of contact predictions in CASP12: Co-evolution and deep learning coming of age. Proteins 86 Suppl 1, 51-66, doi:10.1002/prot.25407 (2018).

46 Fox, N. K., Brenner, S. E. \& Chandonia, J. M. SCOPe: Structural Classification of Proteins--extended, integrating SCOP and ASTRAL data and classification of new structures. Nucleic Acids Res 42, D304-309, doi:10.1093/nar/gkt1240 (2014).

47 Rollins, N. J. et al. 3D protein structure from deep mutation scans. Nat. Genet. (accepted; NG-A50096R1 Marks)

48 Jones, D. T., Singh, T., Kosciolek, T. \& Tetchner, S. MetaPSICOV: combining coevolution methods for accurate prediction of contacts and long range hydrogen bonding in proteins. Bioinformatics 31, 999-1006, doi:10.1093/bioinformatics/btu791 (2015).

49 Wang, S., Sun, S., Li, Z., Zhang, R. \& Xu, J. Accurate De Novo Prediction of Protein Contact Map by Ultra-Deep Learning Model. PLoS Computational Biology 13, e1005324, doi:10.1371/journal.pcbi.1005324 (2017). 
Rohl, C. A., Strauss, C. E., Misura, K. M. \& Baker, D. Protein structure prediction using Rosetta. Methods Enzymol 383, 66-93, doi:10.1016/S0076-6879(04)83004-0 (2004).

$47651 \quad$ Yang, J. et al. The I-TASSER Suite: protein structure and function prediction. Nature Methods 12, 7-8, doi:10.1038/nmeth.3213 (2015).

$47852 \quad$ Poelwijk, F. J., Socolich, M. \& Ranganathan, R. Learning the pattern of epistasis linking genotype and phenotype in a protein. bioRxiv, doi:10.1101/213835 (2017).

53 Firnberg, E. \& Ostermeier, M. PFunkel: efficient, expansive, user-defined mutagenesis. PLoS One 7, e52031, doi:10.1371/journal.pone.0052031 (2012).

54 Wrenbeck, E. E. et al. Plasmid-based one-pot saturation mutagenesis. Nature Methods 13, 928-930, doi:10.1038/nmeth.4029 (2016).

55 Starita, L. M. et al. Massively Parallel Functional Analysis of BRCA1 RING Domain Variants. Genetics 200, 413-422, doi:10.1534/genetics.115.175802 (2015).

56 Starita, L. M. et al. Activity-enhancing mutations in an E3 ubiquitin ligase identified by high-throughput mutagenesis. Proceedings of the National Academy of Sciences 110, E1263-1272, doi:10.1073/pnas.1303309110 (2013).

57 Starr, T. N., Picton, L. K. \& Thornton, J. W. Alternative evolutionary histories in the sequence space of an ancient protein. Nature 5, e16965, doi:10.1038/nature23902 (2017).

60 Bolognesi, B. et al. The mutational landscape of a prion-like domain. bioRxiv, doi:10.1101/592121 (2019). 


\section{Figures} $\rightarrow$ genetic interactions ('epistasis')
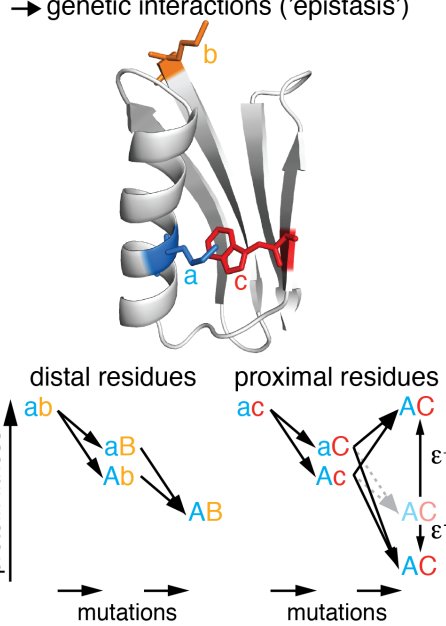
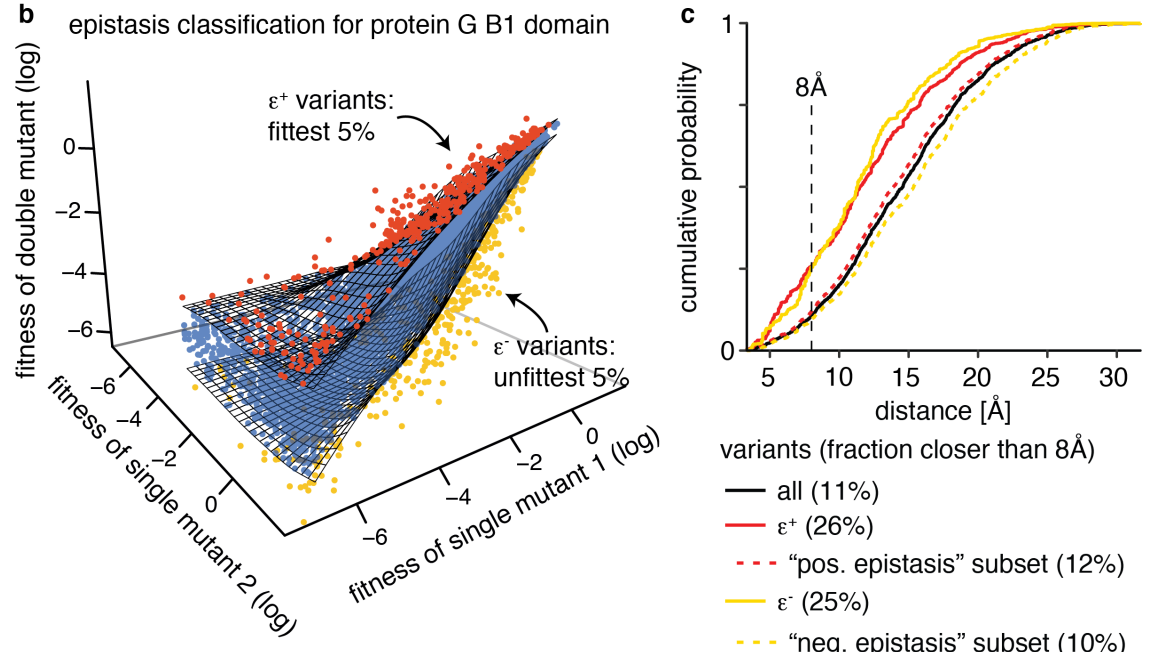

variants (fraction closer than $8 \AA$ )

- all (11\%)

$-\varepsilon^{+}(26 \%)$

- - "pos. epistasis" subset (12\%)

$-\varepsilon^{-}(25 \%)$

"neg. epistasis" subset (10\%)

502 Fig. 1: Extracting epistatic mutational effects from deep mutational

\section{3 scanning of a protein domain}

504

505

506

507

508

509

510

511

512

513

514

515

516

a, Premise: If genetic interactions ('epistasis') are mostly caused by structural interactions then comprehensively quantifying epistatic interactions should suffice to predict a molecule's structure. Structure: protein G B1 domain (PDB entry: 1pga, Ref. ${ }^{61}$ ) with residues a, b, and c colored.

b. Classifying epistatic variants based on deviations from expected fitness (quantile fitness surface approach). Variants with $5 \%$ most extreme fitness values given fitness of their respective single mutants were classified as positive $\left(\mathrm{red}, \varepsilon^{+}\right)$or negative (yellow, $\varepsilon^{-}$) epistatic. Shown is a random sample of $10^{4}$ variants in GB1 domain ${ }^{13}$.

c, Distance distribution of epistatic variants separated by more than 5 amino acids in the linear sequence (minimal side-chain heavy atom distance). Positive and negative epistasis subsets refer to the sets of variants applicable for epistasis analysis (see Supplementary Fig. 1c). All variants, $n=400,647$; positive epistatic variants $\varepsilon^{+}, n=14,127$; positive epistasis subset, $n=$ 315,862 ; negative epistatic variants $\varepsilon^{-}, n=9,837$; negative epistasis subset, $n=208,442$. 
a

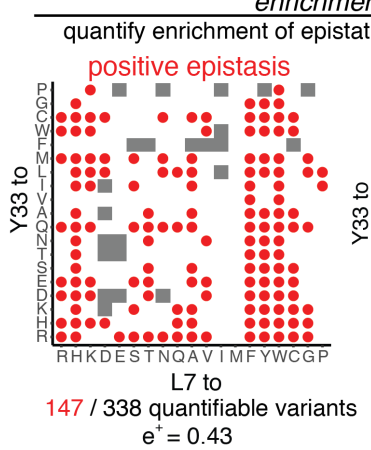

enrichment score

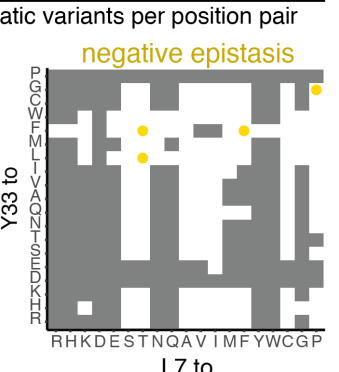

$4 / 155$ quantifiable variants $e^{-}=0.03$

c

correlation score

quantify similarity of epistatic interaction profiles between position pairs

positive epistasis interaction profiles

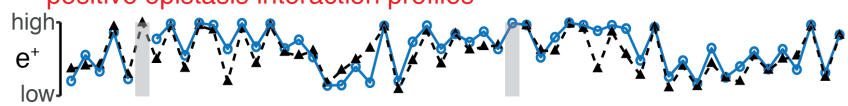

negative epistasis interaction profiles

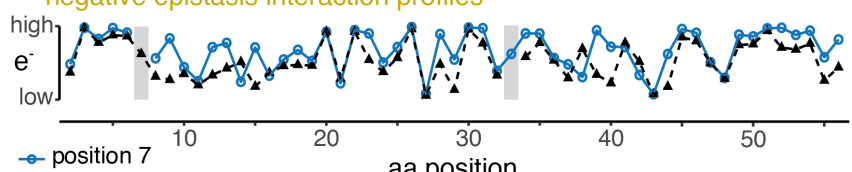

$\rightarrow$ position 7

-A- position 33

d correlation partial correlation correlation score

b top 28 pairs: positive / negative enrichment score epistatic interactions
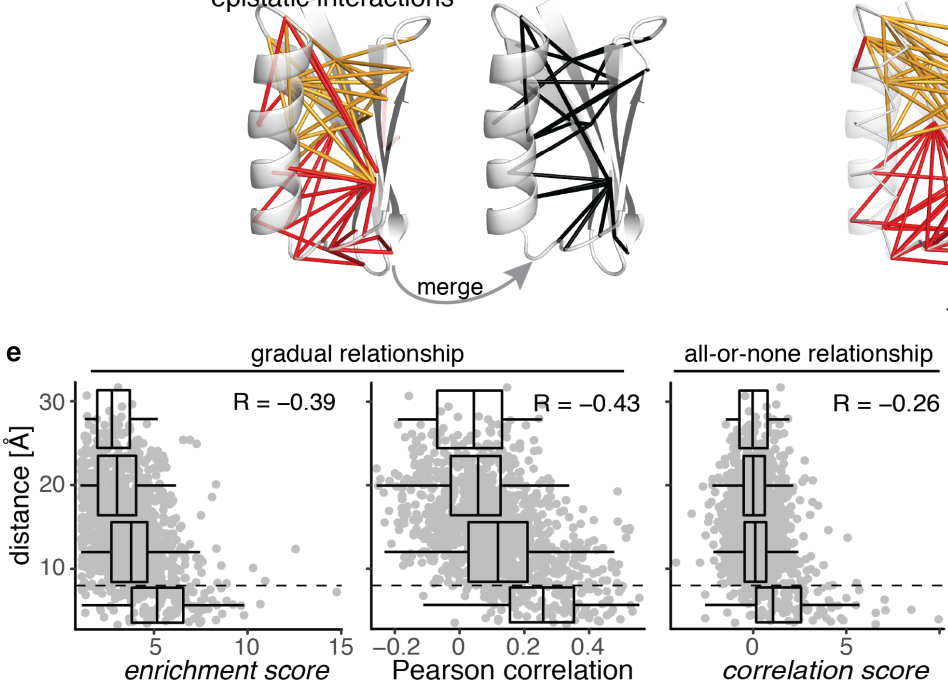

g

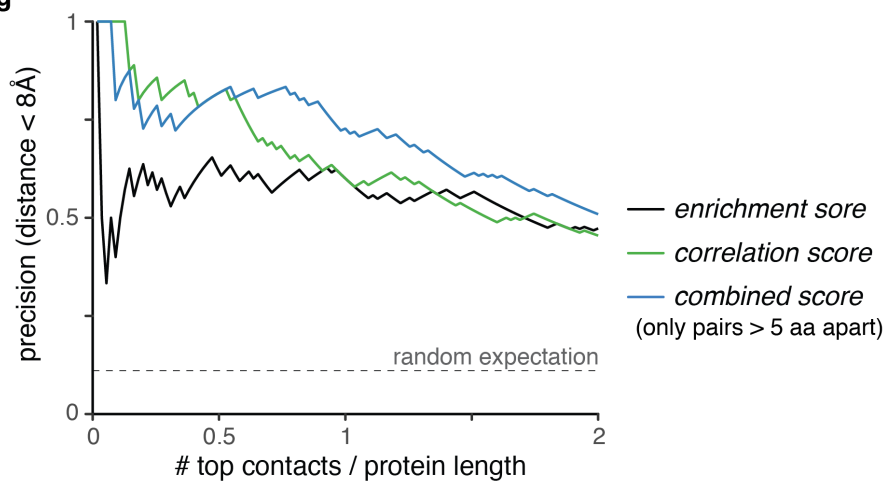

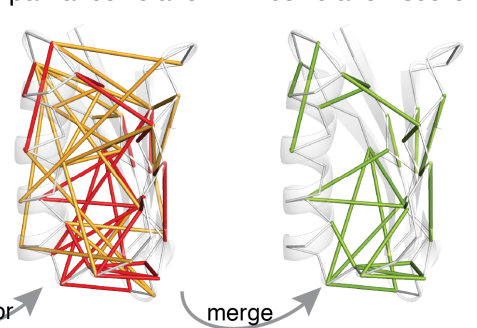

transitive interactions
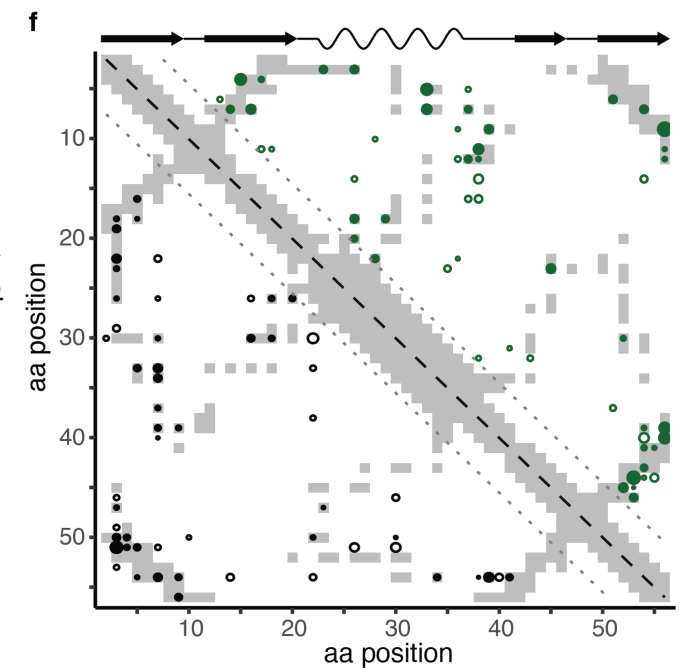

distance scores

- smaller $8 \AA$ - top 55 enrichment score pairs : min - larger $8 \AA ̊$ top 55 correlation score pairs : $\max$ Q

518 Fig. 2: Likelihood of epistatic interactions and correlated interaction profiles 519 predict tertiary structure contacts

520 a, Quantifying enrichment of positive and negative epistatic interactions for position pairs (here 521 positions 7 and 33). Grey shading indicates epistatic interactions are not quantifiable (see 522 Supplementary Fig. 1C-f) 
523 b, Structural distribution of top 28 epistatic interaction pairs (PDB entry 1pga). Left: Pairs with 524 highest positive (red) and negative (yellow) epistatic enrichments. Right: Pairs with highest 525 enrichment scores.

526 c, Example of positive (upper) and negative (lower) epistatic interaction profiles for positions 7 527 and 33 (marked by grey horizontal bars).

528 d, Structural distribution of top 28 pairs with highest positive (red) or negative (yellow) Pearson 529 correlations (left), partial correlations (middle) or correlation scores (right) of interaction profiles.

$530 \mathbf{e}$, Distance of position pairs (> 5aa in linear sequence, $n=1,225$ ) as a function of enrichment 531 scores, merged Pearson correlation of epistasis interaction profiles or correlation scores. 532 Boxplots are spaced in intervals of $8 \AA$; boxes cover $1^{\text {st }}$ to $3^{\text {rd }}$ quartile of the data, with middle 533 bar indicating median, whiskers extend at maximum to 1.5-times the inter quartile range away 534 from the box. Dashed horizontal line indicates $8 \AA$ threshold. Pearson correlation coefficients 535 are indicated.

536 f, Distribution of top 55 position pairs (> 5 aa in linear sequence, indicated by dotted lines) with 537 highest enrichment score (black, lower left triangle) or correlation scores (green, upper right 538 triangle) on contact map of the reference structure (grey shading). Reference secondary 539 structure elements (wave - alpha helix, arrow - beta strand) are shown on top.

$540 \mathrm{~g}$, Precision of interaction scores to predict direct contacts (distance $<8 \AA$ ) as a function of top 541 scoring position pairs. There are 131 direct contacts out of 1,225 pairs ( $>5$ aa in linear 542 sequence), horizontal dashed line indicates random expectation. 


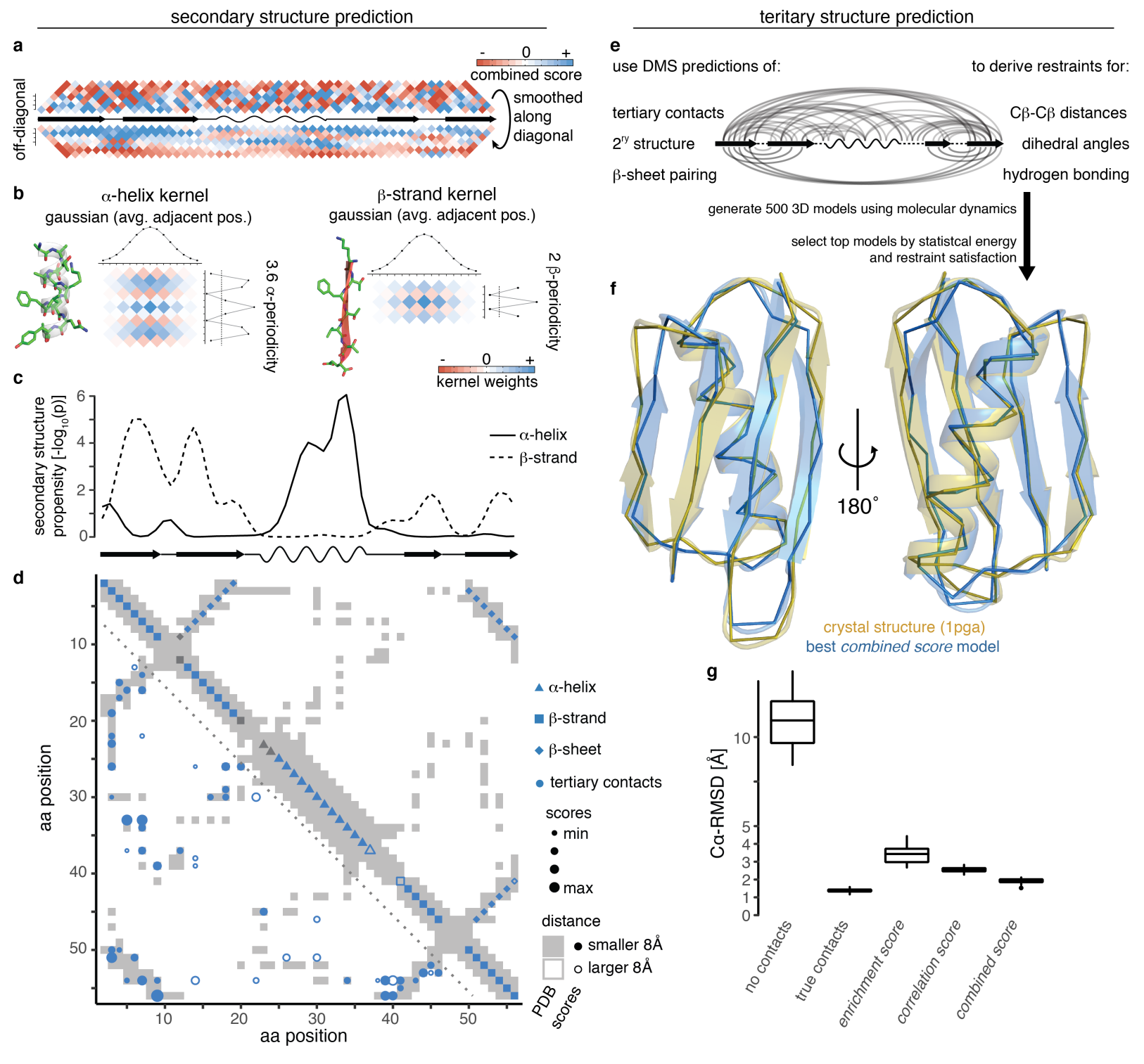

544 Fig. 3: Secondary and tertiary structure prediction from deep mutational

545 scanning data

546 a, Local interactions (above diagonal - raw combined scores up to 7 aa distance in linear 547 sequence, below diagonal - scores smoothed with Gaussian kernel) reveal signatures of 548 secondary structure. Middle line is diagonal of interaction score map (rotated by 45 degrees) 549 and shows secondary structure elements of reference structure.

550 b, 2D kernels with sinusoidal profile to detect stereotypical alpha helical (left, period of 3.6) and 551 beta strand (right, period of 2) interactions and perpendicular Gaussian profile to average over 552 similar interaction patterns in adjacent positions. 
553 c, Secondary structure propensity p-values derived from kernel smoothing (one-sided 554 permutation test, see Methods) in comparison to reference structure secondary structures 555 (wave - alpha helix, arrow - beta strand).

556 d, Structural predictions derived from combined score data compared to reference structure 557 contact map (grey shading). Lower left: Top 55 non-local (>5 aa in linear sequence) tertiary 558 contacts. Upper right: Predicted secondary structure elements. Fill indicates correct prediction.

559 Beta strand predictions are derived by intersection of beta strand propensities (panel c) and 560 beta sheet pairing predictions (Supplementary Fig. 3b,c).

561 e, Scheme for generation of 3D structural models (see Methods for details).

562 f, Overlay of top structural model of protein G B1 domain generated with restraints from 563 combined score (blue) and crystal structure (gold, PDB entry 1pga).

564 g, Accuracy ( $C \alpha$ root-mean-square deviation) of top 5\% structural models ( $n=25)$ generated 565 from interaction score-derived restraints (three right-most columns) compared to reference 566 structure. Left: 'No contacts' - negative control with restraints only for secondary structure 567 (predicted by PSIPRED) ${ }^{62}$. 'True contacts' - positive control with restraints derived from 55 568 random tertiary contacts, secondary structure elements and beta sheet interactions of the 569 reference structure. Boxplots: boxes cover $1^{\text {st }}$ to $3^{\text {rd }}$ quartile of the data, with middle bar 570 indicating median, whiskers extend at maximum to 1.5-times the inter-quartile range away from 571 the box. 


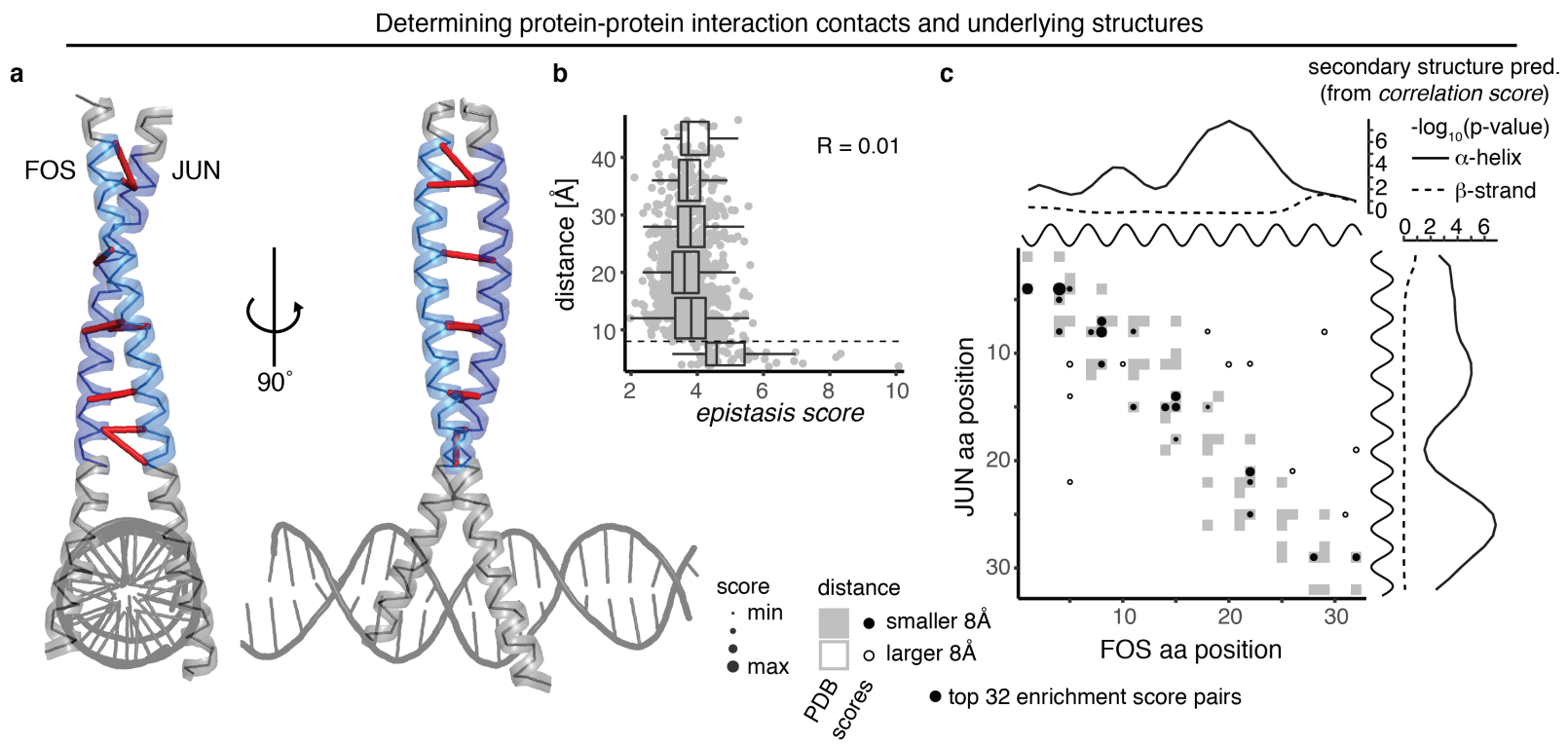

577 Fig. 4: Deep mutagenesis identifies protein-interaction contacts

578 a, Crystal structure of the leucine zipper domains of FOS and JUN with a DNA strand (PDB entry 1 fos). The mutated regions (32 amino acids each) are highlighted in light blue (FOS) and dark blue $(\mathrm{JUN})^{11}$. Top 10 enrichment score pairs are shown with red dashes, note that two interactions between position 8 in FOS and positions 7 and 8 in JUN, as well as three interactions between positions 14 and 15 in FOS and positions 14 and 15 in JUN are hard to distinguish.

b. Distance of position pairs as a function of enrichment scores ( $n=1,024)$. Boxplots are spaced in intervals of $8 \AA$; boxes cover $1^{\text {st }}$ to $3^{\text {rd }}$ quartile of the data, with middle bar indicating median, whiskers extend at maximum to 1.5-times the inter quartile range away from the box. Dashed horizontal line indicates $8 \AA$ threshold. Pearson correlation coefficient is indicated.

c, FOS-JUN trans interaction score map for top 32 position pairs with highest enrichment scores, compared to contact map of known interaction structure (1fos, underlying in grey). Note that protein-protein interaction maps are not symmetric. Shown on top and to the right of the contact map are the known alpha helices (black) as well as the secondary structure propensities

592 derived from correlation scores of FOS and JUN (one-sided permutation test, see also 593 Supplementary Fig. 4a,b). 

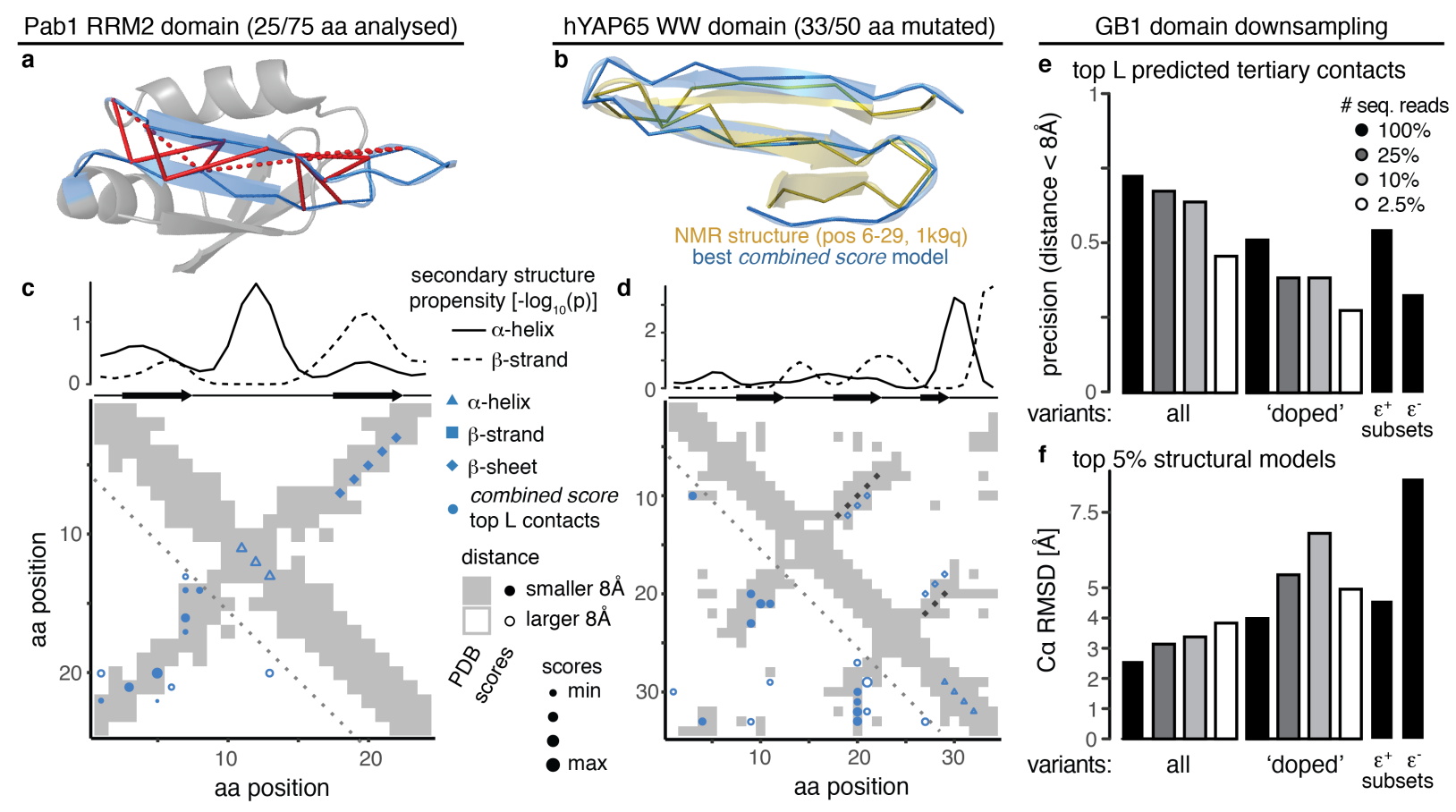

596 Fig. 5: Generality and data requirements for successful protein structure prediction from DMS data

a, Pab1 RRM2 domain (PDB entry 1cvj), the analyzed 25aa segment highlighted in blue. Top 12 combined score position pairs are connected with red lines, solid if distance $<8 \AA$, dashed otherwise.

b, Overlay of top structural model of hYAP65 WW domain (positions 6-29) generated with restraints from combined score (blue) and solution NMR structure (gold, PDB entry 1k9q).

c, Structural predictions derived from combined scores in RRM domain. Upper plot shows secondary structure propensities from kernel smoothing (one-sided permutation test) in comparison to secondary structures in reference. Map shows top 12 combined score position pairs in lower left and secondary structure predictions in upper right triangle, in comparison to 607 reference contact map (grey shading).

d, Structural predictions derived from combined scores in WW domain. Upper plot shows secondary structure propensities from kernel smoothing (one-sided permutation test) in comparison to secondary structures in reference. Map shows top 17 combined score position pairs in lower left and secondary structure predictions in upper right triangle, in comparison to 
612 reference contact map (grey shading). Black diamonds indicate positions of beta sheet pairing 613 in reference.

614 e, Precision of top $L$ combined score position pairs for different down-sampled versions of GB1 615 dataset (in terms of type of variants analysed or sequencing coverage).

616 f, Accuracy $\langle C \alpha-R M S D\rangle$ of top $5 \%$ structural models $(\mathrm{n}=25)$ derived with tertiary contact 617 restraints from down-sampled GB1 datasets compared to reference structure.
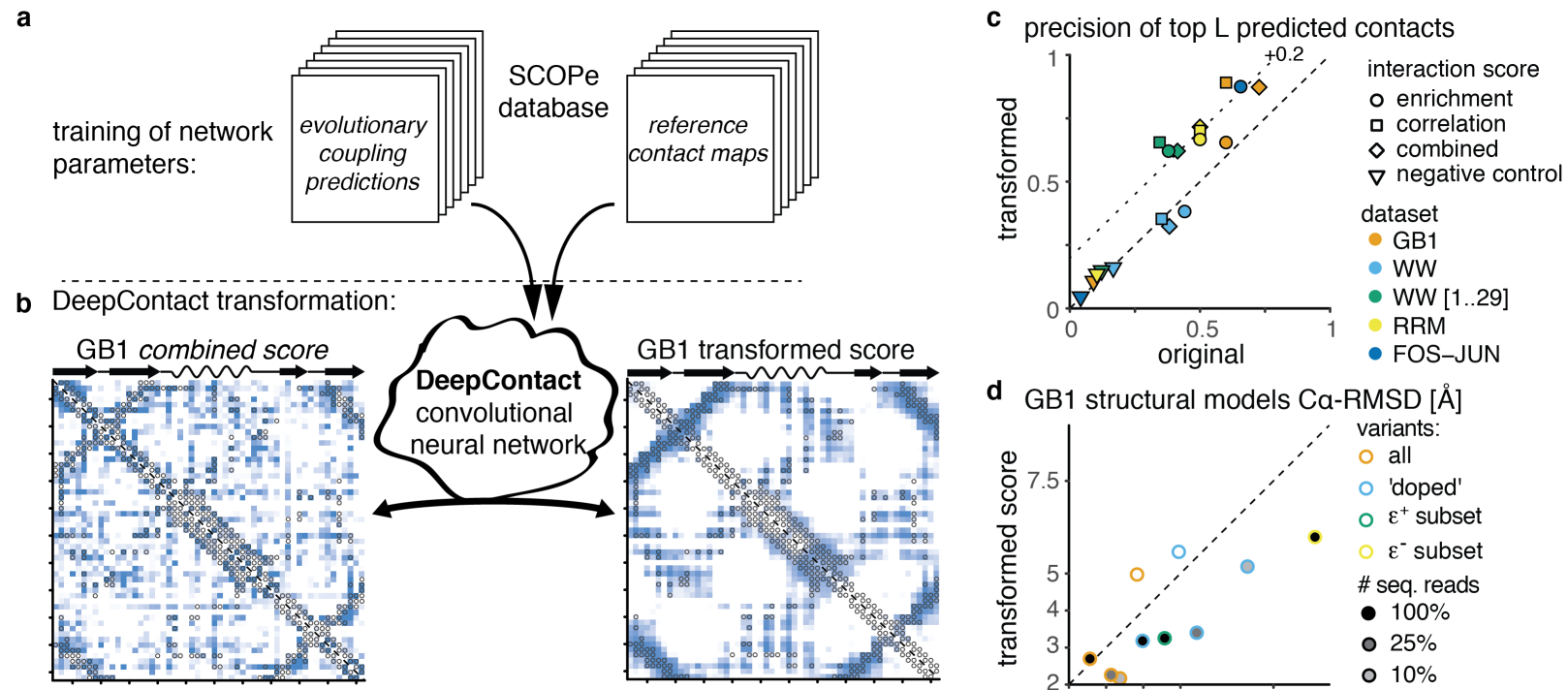

d GB1 structural models Ca-RMSD [Å]

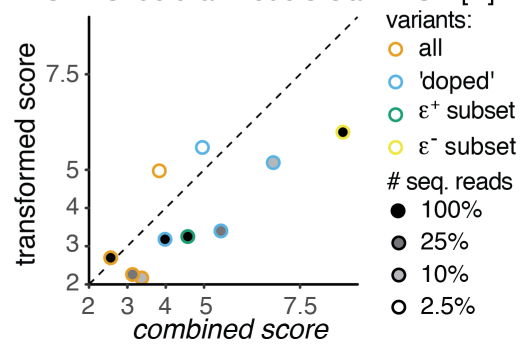

619 Fig. 6: Deep learning improves contact prediction and structural models 620 from deep mutagenesis data

621 a, DeepContact convolutional neural network transforms DMS-derived interaction score maps 622 based on learned structural patterns ${ }^{44}$. The basic DeepContact architecture used here takes as 623 the only input the DMS-derived interaction score map and transforms it based on structural 624 patterns previously learned on an orthogonal and independent training set (in which it compared 625 evolutionary coupling-derived contact predictions with contacts in known structures of 626 representative protein families in the SCOPe database).

627 b, GB1 domain combined score interaction map before (left panel) and after (right panel) 628 transformation with DeepContact convolutional neural network. Heat maps show scores (low 629 white, high - blue). Grey open circles show contacts (distance $<8 \AA$ ) in reference structure. 
630 c, Precision of top $L$ predicted contacts before and after DeepContact transformation. Negative 631 control is average over three random permutations of combined score matrices (in case of FOS632 JUN dataset enrichment score matrices).

633 d, Comparison of accuracy $\langle C \alpha-R M S D\rangle$ of top 5\% GB1 structural models ( $\mathrm{n}=25$ each) with 634 restraints derived either from combined scores or from DeepContact-transformed combined 635 scores for different (down-sampled) GB1 DMS datasets. 


\section{Table 1: Dataset properties}

\begin{tabular}{|l|l|l|l|l|l|l|l|}
\hline Dataset & $\begin{array}{l}\text { Mutated } \\
\text { aa } \\
\text { positions }\end{array}$ & $\begin{array}{l}\text { \% double } \\
\text { mutants }\end{array}$ & $\begin{array}{l}\text { \% doubles } \\
\text { quantifiable }\end{array}$ & $\begin{array}{l}\text { \# input reads } \\
\text { per double } \\
\text { mutant } \\
\text { (median) } \\
\text { epistasis }\end{array}$ & $\begin{array}{l}\text { negative } \\
\text { epistasis }\end{array}$ & $\begin{array}{l}\text { measurement } \\
\text { range } \\
\text { (log fitness } \\
\text { units) }\end{array}$ & $\begin{array}{l}\text { relative } \\
\text { error } \\
\text { (median) }\end{array}$ \\
\hline $\begin{array}{l}\text { Protein G } \\
\text { B1 } \\
\text { domain }\end{array}$ & 55 & 97 & 80 & 55 & 248 & 6 & $2.8 \%$ \\
\hline $\begin{array}{l}\text { WYAP } \\
\text { WW } \\
\text { domain }\end{array}$ & 33 & 10 & 8.3 & 0.8 & 73 & 0.8 & $8.6 \%$ \\
\hline $\begin{array}{l}\text { Pab1 } \\
\text { RRM2 } \\
\text { domain }\end{array}$ & 25 & 11 & 8.3 & 3.9 & 209 & 3.1 & $3.7 \%$ \\
\hline $\begin{array}{l}\text { FOS- } \\
\text { JUN }\end{array}$ & $2 \times 32$ & 43 & 37 & 31 & 124 & 8.6 & $3.6 \%$ \\
\hline
\end{tabular}

${ }^{\$}$ median percentage of all possible double mutants (361 per position pair) that passed read quality thresholds per 638 position pair

$639{ }^{\#}$ median percentage of all possible double mutants (361 per position pair) that passed read quality thresholds and 640 are deemed suitable for epistasis quantification per position pair

641 * summed number of reads across all input replicates for double mutants that passed read quality thresholds

$642+$ measurement range of selection assay: log fitness range between peak of lethal mutants and the wild-type variant

$643 \&$ median error of fitness estimates of double mutant variants relative to measurement range of selection assay 


\section{Methods:}

\section{Datasets and preprocessing}

646 Protein G B1 domain

647 Protein G B1 domain (GB1) deep mutational scanning data were obtained from the

648 Supplementary Information of Olson et al. ${ }^{13}$. The data consist of summed read counts of three 649 replicate experiments assaying the binding affinity of GB1 variants to immunoglobulin $\mathrm{G}$ (IgG).

650 Read frequencies of each single or double mutant variant in input library and output library (after 651 binding affinity assay) were calculated as variant read counts relative to wild-type variant read 652 counts. A variant's fitness was calculated as the natural logarithm of the ratio of output to input

653 read frequency, i.e. $f_{i}=\log \left(\frac{n_{i}^{\text {out }} / n_{w t}^{\text {out }}}{n_{i}^{\text {in }} / n_{w t}^{\text {in }}}\right)$, with $\mathrm{n}$ as read counts, superscripts denoting input or 654 output sequencing library and subscripts denoting variant $i$ or wild-type variant.

655 The standard error of fitness estimates was calculated from read counts under Poissonian 656 assumptions, i.e. $\sigma_{i}=\sqrt{\frac{1}{n_{i}^{\text {in }}}+\frac{1}{n_{i}^{\text {out }}}+\frac{1}{n_{w t}^{\text {in }}}+\frac{1}{n_{w t}^{\text {out }}}}$ (ref. 64). We note that this is a lower bound 657 estimate of the actual error, due to the lack of replicate information.

658 Each measurement assay has a lower measurement limit due to unspecific background effects 659 (Supplementary Fig. 1a). In the case of the IgG-binding assay for GB1, this is presumably 660 mainly due to unspecific carryover on beads ${ }^{13}$. The fitness values derived from the 661 measurement are therefore a convolution of the actual binding affinities to IgG and nonspecific 662 carryover, i.e. $\exp \left(f_{i}^{\text {measured }}\right)=\exp \left(f_{i}^{\text {binding }}\right)+\exp \left(f^{\text {carryover }}\right)$, and fitness values of variants 663 close to the lower measurement limit of the assay are dominated by unspecific carryover 664 effects. The lower measurement limit of the assay was estimated by two approaches that 665 yielded similar estimates. One, from a kernel density estimate of the single mutant fitness 666 distribution ( $R$ function density with parameter bw set to 0.15), where the position of the lower 667 mode of the data corresponded to $f^{\text {carryover }}=-5.85$. Two, from examining the fitness 668 distribution of double mutants with expected fitness lower than -8 log-units, i.e. double mutants 669 resulting from two lethal or nearly lethal single mutant variants, whose fitness values are thus 670 expected to be dominated by background effects. The median of this background fitness 
671 distribution yielded an estimate of $f^{\text {carryover }}=-6.14$. The mean of the two estimates, i.e.

$672 f^{\text {carryover }}=-6(\sim 0.25 \%$ on linear scale $)$ was used for downstream analyses.

$6737 \%$ of double mutant variants were discarded due to too low sequencing coverage in input or

674 output libraries (Supplementary Fig. 1b). That is, variants with 10 or less input read counts were

675 discarded due to too high errors in fitness estimates. Moreover, variants with less than 200 input

676 reads and no output reads were discarded, because it is not possible to determine their fitness.

677 Above 200 input reads, variants without output reads are certain to be dominated by nonspecific

678 carryover effects. These variants were retained and their fitness was calculated by setting their

679 output read count to 0.5 .

680 GB1 down-sampling

681 Down-sampling of the full GB1 dataset was performed in three different ways. First, to down682 sample the sequencing read coverage, each variant's read count was drawn from a binomial 683 distribution with the number of sequencing reads in the full datasets as trials and the target 684 down-sampling rate $(25 \%, 10 \%$ or $2.5 \%)$ as chance of success. Second, in the 'doped' 685 datasets, only amino acid changes created by one nucleotide mutation from the wild-type 686 sequence (ENA entry M12825) were retained. For the read down-sampled and doped datasets 687 (and combinations of both), the analysis workflow for the full dataset was repeated.

688 For the down-sampled datasets taking only positive or negative epistatic information into 689 account, enrichment and correlation scores were calculated from epistatic enrichment matrices 690 and partial correlation matrices of only positive or negative epistasis information. Instead of 691 merging positive and negative matrices and then calculating z-scores, z-scores were calculated 692 with the individual errors from positive or negative epistasis information only. The combined 693 scores (for which results are reported) for each set were then calculated as for the full dataset 694 by summing standardized enrichment and correlation scores.

\section{5 hYAP WW domain}

696 hYAP WW domain data were obtained from Sequence Read Archive (SRA) entry SRP015751 697 (Ref. 43). Paired-end reads were merged with USearch ${ }^{65}$ and merged reads with any base 698 having a Phred base quality score below 20 were discarded. Read counts from the two 699 technical sequencing replicates were merged and read counts for the same amino acid variants 700 with at most one synonymous mutation in one other codon were summed up. The dataset 701 consists of an input library and three output libraries after consecutive rounds of selection in a 
702 phage display assay. Fitness was estimated as the slope of log frequency (variant counts

703 divided by wild-type counts) changes over the rounds of selection experiment ${ }^{43}$. For each

704 variant at each selection step a Poissonian error of $\sigma_{i, x}=\sqrt{\frac{1}{n_{i}^{x}}+\frac{1}{n_{w t}^{x}}}$ was calculated, with $\mathrm{x}$

705 denoting the selection step. Slopes were calculated as weighted straight line least square fits ${ }^{66}$.

706 Comparison of library-wide changes in variant frequencies between selection rounds suggested

707 differential selection pressures across the rounds. We thus applied a non-equidistant spacing of

$7080.6,1.17$ and 1.22 between selection rounds when calculating slopes. Only variants that have

709 more than 10 reads in the input library and at least one read after the first selection were 710 retained for further analysis ( $45 \%$ of constructed double mutants). The lower fitness limit was 711 calculated as the weighted mean fitness of all variants containing STOP codons (-0.78 in log712 fitness units).

\section{Pab1 RRM2 domain}

714 Pab1 RRM2 domain data were obtained from the Supplementary Table 5 of Melamed et al. ${ }^{12}$.

715 Reported variant read enrichment scores were log-transformed to obtain fitness values. Output 716 reads per variant were deduced from the number of input reads times the read enrichment 717 score and used to calculate a Poissonian error of the fitness estimate. Single-mutant count data 718 are not provided and we thus estimated the error of single-mutant fitness estimates to be 0.01 . 719 Lower bound of fitness assay was estimated as weighted mean fitness of all double mutant 720 variants containing STOP codons (-3.1 log-fitness units). In the dataset, three 25 aa segments 721 were mutated independently, and we restricted analysis to the middle segment (position 26-50) 722 containing a significantly number of non-local contacts.

\section{FOS-JUN interaction}

724 Raw count tables were provided by Guillaume Diss ${ }^{11}$. The dataset consists of input and output 725 sequencing libraries after selection for physical interaction between the two proteins in a protein 726 complementation assay in three biological replicates. Per sequencing library, read counts from 727 all synonymous variants were summed up. Only variants that had more than 10 reads in each of 728 the three input libraries were used for further analysis (43\% of double mutants). Per input/output 729 replicate, fitness of each variant was calculated as the log change in frequency compared to the 730 wild-type variant (as for GB1). A Poissonian error for each variant's fitness estimate was 731 derived. Lower measurement bound of fitness assay was estimated as weighted mean fitness of 732 all double STOP mutants variants (-8.6 log-fitness units). A Bayesian estimator of fitness values 
733 was implemented to overcome variant dropout due to a large dynamic range of the fitness

734 assay (see Supplementary Note).

\section{Epistasis classification}

736 Epistasis was calculated from a non-parametric null model - running quantile surfaces - in 737 order to account for nonlinearities close to the lower limit of the fitness assay measurement 738 range, non-specific epistatic behavior resulting from e.g. thermodynamic stability thresholds as 739 well as differential uncertainty of fitness measurements across the fitness landscape, due to 740 lower read counts in the output for low fitness variants (Fig. 1b).

741 First, double mutant fitness values were corrected by subtracting the average local fitness 742 computed using a 2D local polynomial regression (using $R$ function loess with span $=0.2$ ). This 743 was necessary to avoid boundary effects of quantile-based fits in boundary regions with non744 zero slopes. 5th and 95th percentile surfaces were then fit to these residual double mutant 745 fitness values, by computing for each double mutant variant the 5th and 95th percentile of the 746 fitness distribution made up of the $1 \%$ closest neighbors in single mutant fitness space. Double 747 mutant variants with fitness values below the 5th or above the 95th percentile were categorized 748 as negative or positive epistatic, respectively (Fig. 1b).

749 The evaluation of positive or negative epistasis was, however, restricted to specific subsets of 750 the data where measurement errors do not impede epistasis classification (see Supplementary 751 Note and Supplementary Fig. 1c). As a result of these restrictions as well as differences in initial 752 coverage, the number of double mutant variants that can be used to assess positive and 753 negative epistasis varies substantially across position pairs and datasets (see Table 1, 754 Supplementary Figs. 1d-f, 4c and 5a).

\section{Interaction scores}

756 Several interaction scores were derived to estimate which position pairs are in close contact in 757 the tertiary structure (see Fig. 2a,c; and Supplementary Fig. 8 for an overview of the workflow). 758 These scores are based on summarizing epistasis information on the position pair-level and 759 accounting for the uncertainty inherent in the summarized estimates due to differential error of 760 fitness estimates across the measurement range as well as varying numbers of double mutants 761 amenable to epistasis classification (see Table 1, Supplementary Figs. 1d-f, 4c and 5a). To 762 summarize epistasis information on the position pair-level, the fraction of positive or negative 
763 epistatic variants per position pair was calculated (number of epistatic variants divided by the 764 number of variants amenable for epistasis classification, Supplementary Fig. 8, step 5b). 765 Because enrichments with positive and negative epistatic variants per position are anti766 correlated (Supplementary Fig. 2a), positive and negative enrichments were treated separately 767 and only aggregated to derive the final interaction scores. Uncertainty of interaction scores was 768 calculated from a re-sampling procedure where variants fitness values as well as resulting 769 epistatic fractions were drawn from appropriate probability distributions (see Supplementary 770 Note for details and Supplementary Fig. 8, step 5).

771 Enrichment scores, which quantifies how often positions interact epistatically, were derived by 772 merging positive and negative epistatic fractions by weighted averaging, i.e. $\left\langle e_{x y}\right\rangle=$ $773 \frac{\left\langle e_{x y}^{+}\right\rangle * \sigma_{e_{x y}^{+}}{ }^{-2}+\left\langle e_{x y}^{-}\right\rangle * \sigma_{e_{x y}^{-}}{ }^{-2}}{\sigma_{e_{x y}^{+}}{ }^{-2}+\sigma_{e_{x y}^{-}}{ }^{-2}}$, with $\left\langle e_{x y}^{+/-}\right\rangle$as mean epistatic fractions and $\sigma_{e_{x y}^{+/-} \text {as variance of }}$ 774 epistatic fractions across resampling runs. These merged epistatic fractions were further 775 normalized by their uncertainty, i.e. $E_{x y}=\left\langle e_{x y}\right\rangle / \sigma_{x y}$, with $\sigma_{x y}=\left(\sigma_{e_{x y}^{+}}{ }^{-2}+{\sigma_{e_{x y}^{-}}}^{-2}\right)^{-1 / 2}$, to arrive 776 at the final enrichment score (Supplementary Fig. 8, step 6).

777 Correlation scores are derived from the similarity of epistasis interaction profiles between 778 position pairs. The rationale behind this score is that proximal positions in the protein should 779 have similar distances and geometrical arrangements towards all other positions in the protein 780 and should therefore also have similar profiles of epistatic interactions with all other positions. 781 First, a symmetric epistatic fraction matrix (mutated aa positions $\times$ mutated aa positions) for each positive and negative enrichments was constructed (Supplementary Fig. 8, step 5c). 783 Missing values (positions pairs without observed variants) were imputed by drawing a random 784 value from the overall distribution of epistatic fractions. A pseudo-count equal to the first quartile 785 of the epistatic fraction distribution was added to all matrix entries. Diagonal elements (epistatic 786 fractions of a position with itself) were set to 1 . The matrix values were transformed by the 787 natural logarithm and for each pair of columns the Pearson correlation coefficient was 788 calculated to arrive at the correlation matrix (step $5 \mathrm{~d}$ ). The correlation matrix was regularized 789 using a shrinkage approach ${ }^{67}$, in order to minimize the mean-squared error between estimated 790 and true correlation matrix and obtain a positive definite and well-conditioned correlation matrix 791 suitable for inversion (R package corpcor). Next, partial correlations of epistatic interaction 792 profiles between each position pair were calculated by inverting the regularized correlation 793 matrix and normalizing each off-diagonal entry of the inverted matrix by the geometric mean of 
794 the two respective diagonal entries, i.e. $a_{x y}^{+}=\frac{r_{x y}^{-1}}{\sqrt{r_{x x}^{-1} * r_{y y}^{-1}}}$, with $r_{x y}^{-1}$ as the $(\mathrm{x}, \mathrm{y})$-entry of the 795 inverted correlation matrix (Supplementary Fig. 8, step 5d). We note that this approach is similar 796 to how mean-field approaches can help discriminate direct from indirect evolutionary couplings 797 in multiple sequence alignments ${ }^{24,30,68}$. Equivalent to the enrichment score, positive and 798 negative partial correlation estimates were merged by calculating weighted averages of their 799 mean estimates across re-sampling runs, with weights as the inverse variances across 800 resampling runs, i.e. $\left\langle a_{x y}\right\rangle=\frac{\left\langle a_{x y}^{+}\right\rangle *{\sigma_{a_{x y}^{+}}}^{-2}+\left\langle a_{x y}^{-}\right\rangle * \sigma_{a_{x y}^{-}}{ }^{-2}}{\sigma_{a_{x y}^{+}}{ }^{-2}+\sigma_{a_{x y}^{-}}{ }^{-2}}$, and the final correlation score normalized 801 by the combined uncertainty, $A_{x y}=\left\langle a_{x y}\right\rangle / \sigma_{x y}$, with $\sigma_{x y}=\left(\sigma_{a_{x y}^{+}}{ }^{-2}+{\sigma_{a_{x y}^{-}}}^{-2}\right)^{-1 / 2}$ (step 6).

802 Finally, a combined score was derived by summing the standardized enrichment and correlation 803 scores, i.e. $C_{x y}=\frac{E_{x y}-\langle E\rangle}{\sigma_{E}}+\frac{A_{x y}-\langle A\rangle}{\sigma_{A}}$, in order to prioritize position pairs that are enriched for 804 epistatic interactions and have similar epistasis profiles. We note that this is a naïve approach to 805 combining the information from these two complementary sources, and surely more 806 sophisticated approaches that further improve proximity estimates can be developed.

\section{Protein distance metrics}

808 The minimal distance between side chain heavy atoms of two residues (in case of glycine, C $\alpha$ ) 809 was used as the distance metric. A direct contact was defined as minimal side-chain heavy 810 atom distance $<8 \AA$. Only position pairs with linear sequence separation greater than 5 aa were considered when evaluating tertiary contact predictions. Evaluating contact predictions only on 812 side-chain heavy atom distances instead of all heavy atoms increases true positive rates over 813 random expectation, thus suggesting that epistatic interactions are mostly mediated by 814 structural interactions of amino acid side-chains (Supplementary Fig. 7).

815 Reference structures used as comparison were

- GB1 domain: PDB entry 1pga, X-ray diffraction structure ${ }^{61}$

- WW domain: PDB entry $1 \mathrm{k} 9 \mathrm{q}$, solution NMR structure ${ }^{69}$

- RRM domain: PDB entry 1cvj (chain A), X-ray diffraction structure of human Pab1 (Ref. 70); note that the central section of the yeast RRM domain analyzed is one nucleotide longer than the corresponding homologous region in the human RRM domain. We thus 
arbitrarily removed position 14 (in the loop region, as done in Melamed et al. ${ }^{12}$ ) when comparing the DMS-derived predictions to the human Pab1 structure.

- FOS-JUN interaction: PDB entry 1 fos (chains $E$ and F), X-ray diffraction structure ${ }^{71}$

824 We found that precision or accuracy calculated against other reference structures differed only 825 marginally, thus we have limited reporting to the aforementioned PDB entries.

\section{Secondary structure prediction}

827 Secondary structure elements were predicted using a 2D kernel smoothing approach on the 828 interaction score matrices (Fig. 3a-c). For a given aa position in the linear chain (on the diagonal 829 of the interaction score matrix), the perpendicular dimension of the kernels define how 830 interactions with adjacent positions (off-diagonal entries close to the diagonal) should be 831 integrated given the interaction patterns expected from the stereotypical periodicities of 832 secondary structures, i.e. 3.6 aa in alpha helices and 2 aa in beta strands. Moreover, the 833 diagonal dimension of the kernels average the stereotypical interaction patterns of secondary 834 structures across several adjacent positions. Similar, modified beta strand kernels were used to 835 detect beta sheet interactions for all pairs of positions. Significance of secondary structure 836 element predictions was assessed from a permutation test, where kernel smoothing was 837 performed on $10^{4}$ randomly permutated interaction score maps. For more details on secondary 838 structure predictions see the Supplementary Note.

\section{Protein structure prediction}

840 Protein structures were modeled ab initio with structural restraints derived from the deep 841 mutational scanning data using simulated annealing molecular dynamics (XPLOR-NIH modeling 842 suite $^{42}$, see Supplementary Note for details).

\section{DeepContact learning}

844 DeepContact software was obtained from GitHub (https://github.com/largelymfs/deepcontact) ${ }^{44}$. 845 We are grateful to Yang Liu and Jian Peng for also making - without any hesitation - their basic 846 DeepContact network architecture available on their GitHub repository and helping us with the 847 implementation. The DeepContact architecture used here only takes one 2D input of predicted 848 contact scores and returns a 2D map of transformed scores (denoted as "DeepContact 849 CCMPred only" in Ref. ${ }^{44}$ and described in the first paragraph of the result section therein). The 
DeepContact architecture employed came with a pre-trained network model that had been trained by comparing tertiary contact predictions from correlated evolution (using CCMpred ${ }^{74}$ ) to

852 experimentally determined structures of proteins in the 40\% homology filtered ASTRAL SCOPe

8532.06 dataset (see GitHub repository and Liu et al. ${ }^{44}$ ). Because CCMpred scores ${ }^{74}$ are 854 distributed in the range of 0 to 1 , deep mutational scanning derived interaction scores were pre855 normalized to range between 0 and 1 before providing them as an input to DeepContact. As 856 negative control, we created for each dataset three random permutations of combined score 857 matrices (while preserving matrix symmetry; in case of FOS-JUN dataset non-symmetric 858 enrichment score matrices were permutated), which were transformed by the DeepContact 859 algorithm. These control datasets show no increased precision over random expectation (Fig. $8606 c)$.

\section{Code availability}

862 Paired-end sequencing reads were merged with USearch v10.0.240. Data were analyzed with 863 custom scripts written and executed in $\mathrm{R}$ programming language, version 3.4.3. Structural 864 simulations were performed with Xplor-NIH modeling suite version 2.46. TM-Score (update 865 2016/03/23) was used to evaluate accuracy of structural models. PSIPRED v3.3 was used to 866 predict secondary structure elements from amino acid sequence. PyMOL v1.8.6.0 was used to 867 visualize protein structures. All custom scripts needed to repeat the analyses are available at 868 https://github.com/jschmiedel/DMS2structure.

\section{Data Availability}

870 No primary data were generated in this study. Data sources are listed in the Methods section at 871 appropriate places. Processed interaction scores for all datasets are included in Supplementary 872 Table 1. All intermediate steps of data processing can be recapitulated with the scripts provided 873 at https://github.com/jschmiedel/DMS2structure.

\section{Reporting Summary}

875 Further information on study design is available in the Life Sciences Reporting Summary linked 876 to this article. 


\section{Methods-only References}

$87861 \quad$ Gallagher, T., Alexander, P., Bryan, P. \& Gilliland, G. L. Two crystal structures of the B1 immunoglobulin-binding domain of streptococcal protein $\mathrm{G}$ and comparison with NMR Biochemistry 33, 4721-4729 (1994).

62 Jones, D. T. Protein secondary structure prediction based on position-specific scoring matrices. Journal of Molecular Biology 292, 195-202, doi:10.1006/jmbi.1999.3091 (1999).

63 The PyMOL Molecular Graphics System, V. S., LLC. The PyMOL Molecular Graphics

64 Rubin, A. F. et al. A statistical framework for analyzing deep mutational scanning data.

65 Edgar, R. C. Search and clustering orders of magnitude faster than BLAST. Bioinformatics 26, 2460-2461, doi:10.1093/bioinformatics/btq461 (2010).

66 Barlow, R. Statistics : a guide to the use of statistical methods in the physical sciences.

67 Schäfer, J. \& Strimmer, K. A shrinkage approach to large-scale covariance matrix estimation and implications for functional genomics. Statistical applications in genetics

68 Stein, R. R., Marks, D. S. \& Sander, C. Inferring Pairwise Interactions from Biological

69 Pires, J. R. et al. Solution structures of the YAP65 WW domain and the variant L30 K in complex with the peptides GTPPPPYTVG, N-(n-octyl)-GPPPY and PLPPY and the

70 Deo, R. C., Bonanno, J. B., Sonenberg, N. \& Burley, S. K. Recognition of polyadenylate RNA by the poly(A)-binding protein. Cell 98, 835-845 (1999).

72 Adhikari, B., Bhattacharya, D., Cao, R. \& Cheng, J. CONFOLD: Residue-residue contact-guided ab initio protein folding. Proteins 83, 1436-1449, doi:10.1002/prot.24829 (2015).

73 Zhang, Y. \& Skolnick, J. Scoring function for automated assessment of protein structure template quality. Proteins 57, 702-710, doi:10.1002/prot.20264 (2004).

74 Seemayer, S., Gruber, M. \& Söding, J. CCMpred--fast and precise prediction of protein residue-residue contacts from correlated mutations. Bioinformatics 30, 3128-3130, doi:10.1093/bioinformatics/btu500 (2014). 